IAU Colloquium 164: Radio Emission from Galactic and Extragalactic Compact Sources

ASP Conference Series, Vol. 144,1998

J. A. Zensus, G. B. Taylor, E' J. M. Wrobel (eds.)

\title{
Constraints on the Evolution of Powerful Radio Sources from GPS and CSS Sources
}

C. P. O'Dea

Space Telescope Science Institute, Baltimore, MD 21218, U.S.A.

Abstract. I present the results of a study of the global properties of combined complete samples of GPS and CSS sources. I discuss the constraints the results place on models for the evolution of these sources.

\section{Introduction}

The GHz-peaked spectrum (GPS; O'Dea, Baum, \& Stanghellini 1991) and compact steep-spectrum (CSS; Fanti et al. 1990) radio sources are powerful but compact radio sources whose spectra are generally simple and convex with peaks near $1 \mathrm{GHz}$ and $100 \mathrm{MHz}$ respectively. The GPS sources are entirely contained within the extent of the narrow line region $(\lesssim 1 \mathrm{kpc})$ while the CSS sources are contained entirely within the host galaxy ( $\lesssim 20 \mathrm{kpc})$.

GPS and CSS sources are important because (1) they probe the NLR and ISM of the host galaxy and (2) they may be the younger stages of powerful largescale radio sources-giving us insight into radio source genesis and evolution.

\section{Global Properties of GPS and CSS Sources}

Possible scenarios for the evolution of GPS and CSS sources into larger powerful radio sources have been discussed by Carvalho (1985), Hodges \& Mutel (1987), Fanti et al. (1995), Begelman (1996) and Readhead et al. (1996). If the GPS and CSS sources do evolve, then their global properties must constrain that evolution. O'Dea \& Baum (1997) have examined the global properties of two well defined samples-the GPS sample of Stanghellini et al. (1996) and the CSS sample of Fanti et al. (1990). O'Dea and Baum find the following. The distribution of rest-frame turnover frequency extends above $10 \mathrm{GHz}$. This reveals the presence of a previously unsuspected population of sources which peak at high frequencies (see also de Vries et al. 1997). At high redshifts $z \geq 2$ the rest frame turnover occurs above $1 \mathrm{GHz}$. If this is not due to a selection effect it suggests that at high redshifts the sources inhabit regions of high ambient density.

There is a strong anticorrelation between rest-frame turnover frequency $\left(\nu_{m}\right)$ and linear size $(L S)$ of the form $\nu_{m} \propto L S^{-0.65}$. The continuity on this plane suggests that GPS and CSS sources are related either by evolution or by the mechanism for the turnover or both.

The GPS and CSS sources in these samples have similar radio power, and although they span a large range in linear size, there is no correlation between radio power and linear size. The projected linear sizes of the GPS and CSS sources do not appear to vary with redshift. The quasars and galaxies in these samples have similar distributions of projected linear size. Thus the quasars do not appear to be strongly foreshortened version of the galaxies. 


\section{Constraints on Radio Galaxy Evolution}

O'Dea and Baum compare the the GPS and CSS sources with the LRL 3CR classical doubles in the redshift range $0.2 \lesssim z \lesssim 1.0$. The GPS and CSS sources in these bright samples would be members of the LRL 3CR if their spectra did not turn over. The number per bin of log size is roughly constant for the GPS and CSS sources while for the LRL 3CR classical doubles the number increases with increasing size as $N(L S) \propto L S^{0.4}$. These results imply that the GPS and CSS sources may evolve in a qualitatively different way than the LRL 3CR classical doubles. One possibility is that some of the GPS and CSS sources will not evolve into classical doubles and instead might be transient or frustrated sources. Alternately, the GPS and CSS sources, due to their interaction with the ISM of the host galaxy, evolve differently than predicted by simple self similar models.

Assuming that all (or nearly all) the GPS and CSS sources are progenitors of larger sources O'Dea and Baum consider the implications for evolution of the GPS and CSS sources and their relationship to the LRL 3CR classical doubles. A simple model where the sources evolve with constant velocity and constant radio luminosity is ruled out. If the sources evolve with constant velocity, the radio luminosity must decrease with linear size roughly as $L_{\text {radio }} \propto L S^{-0.5}$. This strong luminosity evolution implies that the GPS and CSS sources evolve into sources which are less luminous than the LRL classical doubles, and some sources may evolve into FR1 sources.

Acknowledgments. I am grateful to Carlo Stanghellini for his excellent work on the GPS complete sample. I have benefited from conversations with Stefi Baum, Wim de Vries, Tony. Readhead, Mitch Begelman, Geoff Bicknell, and Greg Taylor.

\section{References}

Begelman, M. C. 1996. in Cygnus A: Study of a Radio Galaxy, eds. C. Carilli, \& D. Harris (Cambridge: Cambridge University Press), 209-214.

Carvalho, J. C. 1985. MNRAS, 215, 463-471.

de Vries, W. H., Barthel, P. D., \& O’Dea, C. P. 1997. $A \& A$, 321, 105-110.

Fanti, R., et al. 1990. $A \mathcal{B} A, 231,333-346$.

Fanti, C., et al. 1995. $A \& A, 302,317-326$.

Hodges, M. W., \& Mutel, R. L. 1987. In Superluminal Radio Sources, eds. J. A. Zensus, \& T. J. Pearson (Cambridge: Cambridge University Press), 168-173.

O'Dea, C. P., Baum, S. A., \& Stanghellini, C. 1991. ApJ, 380, 66-77.

O'Dea, C. P., \& Baum, S. A. 1997. AJ, 113, 148-161.

Readhead, A. C. S., et al. 1996b. ApJ, 460, 634-643.

Stanghellini, C., et al. 1996. in Second Workshop on Gigahertz Peaked Spectrum and Compact Steep Spectrum Radio Sources, eds. Snellen, I. A. G., Schilizzi, R. T., Röttgering, H. J. A., \& Bremmer, M. N. (Leiden: JIVE), 4-13. 